\title{
Variability of Neutral-Position Casting of the Foot
}

\author{
Vivienne Chuter, BPod(Hons)* \\ Craig Payne, DipPod, MPH* \\ Kathryn Miller, BPod*
}

Neutral-position casting of the foot is used for the manufacture of functional foot orthoses, and an accurate cast is widely assumed to be a prerequisite for a good orthotic device. The primary aim of this study was to determine the variability of casting between inexperienced and experienced clinicians and the variability of one experienced clinician taking multiple casts. Ten inexperienced and ten experienced clinicians took a cast of the right foot of a single subject, and a single experienced clinician took ten casts of the same foot. The frontal plane forefoot-to-rearfoot relationship of each cast was determined, and no difference was found in the mean and variances among the three groups. The range of the forefoot-to-rearfoot relationship across all groups was from $10.0^{\circ}$ everted to $6.5^{\circ}$ inverted, indicating that there is a wide range in neutralposition casting of the foot. As outcome studies have reported the successful outcomes of functional foot orthoses, this wide variability may not necessarily be a problem. (J Am Podiatr Med Assoc 93(1): 1-5, 2003)

The management of biomechanical misalignment of the lower extremity and the associated pathologic symptoms often involves the use of foot orthotic therapy. Orthotic prescriptions are aimed at placing the foot in such a position as to encourage as near normal foot alignment and function as possible. Plaster casts of the foot are the most frequent method used to prepare positive casts or models of the foot that the orthoses are molded on.

The foot can be casted weightbearing or nonweightbearing and can be held in many different alignments or positions. Placing the foot in different alignment positions for casting will have implications for the plantar surface contours ${ }^{1}$ and the position that the resultant foot orthosis will place the foot in. For example, significant differences have been reported in the frontal plane forefoot-to-rearfoot relationship between a weightbearing and a nonweightbearing cast. $^{2,3}$

*Department of Podiatry, School of Human Biosciences, La Trobe University, Melbourne, Australia.

Corresponding author: Craig Payne, DipPod, MPH, Department of Podiatry, School of Human Biosciences, La Trobe University, Bundoora, Victoria 3083, Australia.
Most of the foot orthotic therapy used today involves the foot being casted in a nonweightbearing position, with the subtalar joint held in its neutral position and the midtarsal joint in its maximally pronated position. ${ }^{4}$ Root et $\mathrm{al}^{5}$ first introduced this neutral-position casting for the manufacture of functional foot orthoses. The aim of casting in this position is to construct an accurate replica of the existing structural anomalies by capturing the forefoot-to-rearfoot relationship with the foot in its optimal functional position. This method allows abnormalities within the foot to be measured and quantified and forms the basis of the orthotic prescription. The completed functional foot orthotic device should then support the foot in its ultimate functional position, specifically, placing the foot in a neutral subtalar joint position during midstance. ${ }^{6,7}$ The resultant ability of functional foot orthoses to control the motion of the foot about the assumed ideal position of the subtalar joint is thought to depend on the true forefoot-to-rearfoot relationship captured accurately by the negative cast. 4 , 7 To achieve this, the foot placed in a position for casting with the subtalar joint held in a neutral 
position and the midtarsal joint "locked" as a normal foot is assumed to be in midstance. ${ }^{4}$

Root et $\mathrm{al}^{8}$ describe the subtalar joint neutral position as a position in which the joint is neither pronated nor supinated, and it is generally determined clinically by palpating the congruency of the talar head proximal to the navicular. They define the midtarsal joint as being locked when it is maximally pronated about both its oblique and longitudinal axes, and the position is clinically determined by applying a dorsiflexory force to the lateral column of the foot. It is widely assumed that the achievement of these two theoretical constructs-the subtalar joint neutral position and the maximally pronated midtarsal joint - during casting is the essential first step in the manufacture of functional foot orthoses. , $, 6,7$

The primary aim of this study was to determine the variability of neutral-position negative casting of the foot within and between experienced and inexperienced clinician groups to see whether variability decreases with experience and a single clinician taking multiple casts of the same foot. A secondary aim was to compare plaster casts of the same foot taken with the midtarsal joint pronated and supinated to determine whether one position provides less variability.

\section{Materials and Methods}

The study was approved by the Faculty of Health Sciences Human Ethics Committee at La Trobe University in Melbourne, Australia, and informed consent was obtained from all participants. Ten students in the final year of their podiatric medical degree program each took a neutral-position negative cast of the right foot of one subject. Ten experienced clinicians took a neutral-position negative cast of the right foot of the same subject. Experience was defined as taking a minimum of ten negative cast impressions per month for at least the previous 12 months. Participants were instructed to cast the foot with the subtalar joint in its neutral position and the midtarsal joint maximally pronated or locked, by their preferred method. A single experienced clinician also took ten casts of the right foot of the same subject. The same clinician was then instructed to take five additional casts with the subtalar joint in its neutral position and the midtarsal joint pronated about its longitudinal axis and supinated about its oblique axis by applying an adductory force to the lateral forefoot during casting. Another five casts were taken with the oblique axis pronated by applying an abductory force to the forefoot during casting.

The negative casts were then filled, and the positive casts were used for evaluation of the frontal plane forefoot-to-rearfoot relationship captured in the cast. Two researchers examined each cast together to agree on a vertical posterior calcaneal bisection line. An ethyl vinyl acetate wedge was constructed and placed under the medial or lateral forefoot until it was agreed that a bisection of the calcaneus was in a vertical position. A set square was then used to mark the bisection with ink. Two different types of measurements were performed to determine the frontal plane forefoot-to-rearfoot relationship. For the first, an inclinometer was used to measure the posterior bisection of the calcaneus as inverted, everted, or perpendicular when the cast was resting on a flat surface (Fig. 1). The angle measured reflects the frontal plane forefoot-to-rearfoot relationship in the cast. For the second type of measurement, wedging was then placed under the medial or lateral forefoot to move the posterior calcaneal bisection to vertically align with a set square. Using measurement calipers, the height (in millimeters) of the wedge at the edge of the cast was measured (Fig. 2). Each measurement was taken and recorded separately by the same two researchers (V.C. and K.M.) on two separate occasions. The researchers were blinded to the identity of the clinician who had taken the cast. The mean of the values obtained by each researcher on the two occasions was used for the analysis. Intraclass correlation coefficients ${ }^{9}$ (ICCs) were calculated for the reliability of measurements. Pearson's $r$ was calculated to establish the correlation between the millimeters of elevation of the forefoot when the calcaneal bisection was vertical and the angle of the calcaneal bisec-

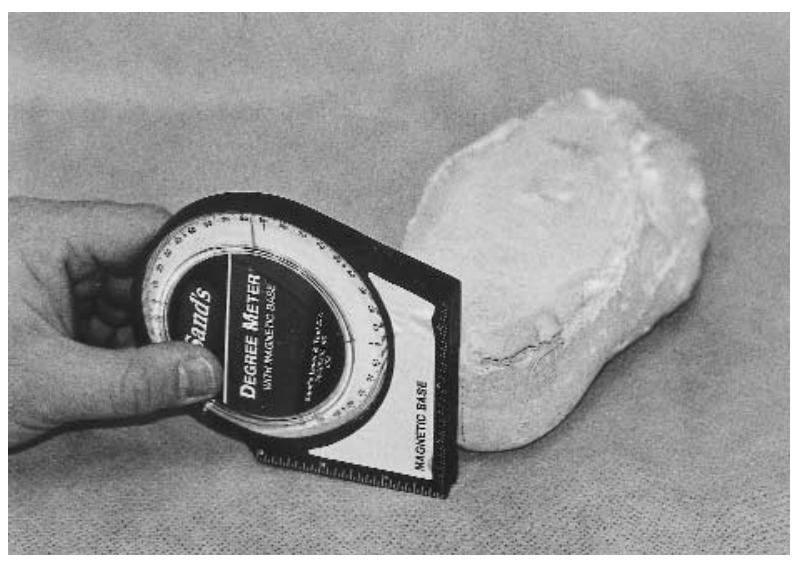

Figure 1. An inclinometer was used to measure the posterior bisection of the calcaneus with the cast resting on a flat surface to determine the forefoot-to-rearfoot relationship. 


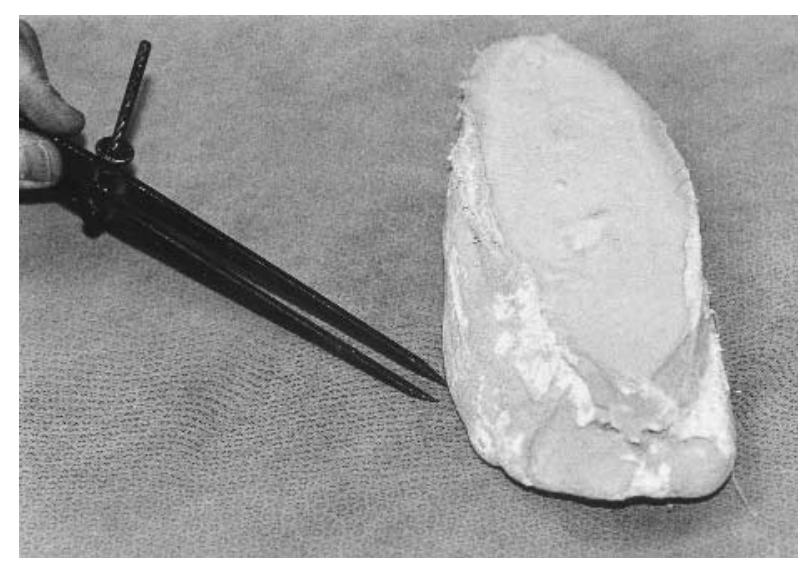

Figure 2. Calipers were used to measure the height of the medial or lateral side of the forefoot of the surface while the posterior bisection was held vertical to determine the frontal plane forefoot-to-rearfoot relationship.

tion when the cast was resting on a flat surface. The differences among the three groups were analyzed using the Kruskal-Wallis test for comparison of means. An F test was used to compare the variability among the three groups of casts. The mean values for the five supinated oblique axis casts and the five pronated oblique axis casts were compared using the Mann-Whitney test.

\section{Results}

The mean \pm SD frontal plane forefoot-to-rearfoot relationship determined by the angle of the posterior bisection of the calcaneus was $3.0^{\circ} \pm 5.0^{\circ}$ everted (range, $10.0^{\circ}$ everted to $2.2^{\circ}$ everted) for the inexperienced group, $1.2^{\circ} \pm 3.3^{\circ}$ everted (range, $7.3^{\circ}$ everted to $3.7^{\circ}$ inverted) for the experienced group, and $0^{\circ} \pm 3.2^{\circ}$ perpendicular for the single clinician (range, $5.7^{\circ}$ everted to $6.5^{\circ}$ inverted). Using the height of the medial or lateral side of the forefoot when the calcaneal bisection was held vertical, for the inexperienced group, the lateral side of the forefoot was a mean $\pm \mathrm{SD}$ of $4.4 \pm 5.0 \mathrm{~mm}$ off the ground (range, $9.3 \mathrm{~mm}$ lateral to $5.9 \mathrm{~mm}$ medial); for the experienced group, the lateral side of the forefoot was $2.7 \pm 5.7 \mathrm{~mm}$ off the ground (range, $8.2 \mathrm{~mm}$ lateral to $6.0 \mathrm{~mm}$ medial); and for the single clinician, the forefoot was $0.5 \pm 5.3 \mathrm{~mm}$ off the ground on the medial side (range, $6.5 \mathrm{~mm}$ lateral to $5.7 \mathrm{~mm}$ medial). The correlation between the overall mean degrees of the calcaneal bisection and mean medial or lateral height of the forefoot off the ground was 0.93 . The type $(3,1)$ ICC $^{9}$ for the measure- ments was 0.99 for the height of the forefoot and 1.0 for the measurement of the calcaneal bisection.

Results of the Kruskal-Wallis test indicated that there was no difference among the three groups in the mean frontal plane forefoot-to-rearfoot relationship as measured by the calcaneal bisection $(P=.15)$ or the height of the forefoot $(P=.17)$. An $\mathrm{F}$ test for equality of variances showed no difference in variability among the groups in either height of the forefoot $(P=.31)$ or angle of the calcaneal bisection $(P=.35)$.

For the five casts with the forefoot adducted (pronated about the oblique axis), the mean \pm SD forefoot-to-rearfoot relationship measured by the calcaneal bisection was $1.9^{\circ} \pm 5.0^{\circ}$ everted (range, $7.1^{\circ}$ everted to $4.2^{\circ}$ everted) and measured by the height of the forefoot was $4.1 \pm 4.7 \mathrm{~mm}$ off the ground on the lateral side (range, $4.8 \mathrm{~mm}$ lateral to $2.5 \mathrm{~mm}$ medial). For the five casts with the forefoot abducted (supinated about the oblique axis), the mean $\pm \mathrm{SD}$ forefoot-to-rearfoot relationship measured by the calcaneal bisection was $2.0^{\circ} \pm 1.8^{\circ}$ everted (range, $3.5^{\circ}$ everted to perpendicular) and measured by the height of the forefoot was $3.4 \pm 2.7 \mathrm{~mm}$ off the ground on the lateral side (range, $6.1 \mathrm{~mm}$ lateral to $0.5 \mathrm{~mm}$ medial). The Mann-Whitney test for differences of means found no significant differences between the means of the two groups for the angle of the calcaneal bisection $(P=.9)$ and the height of the forefoot $(P=.4)$.

\section{Discussion}

There are a number of variations for taking neutralposition negative cast impressions of the foot, all with the aim of creating an accurate replica of the forefoot-to-rearfoot relationship. ${ }^{10}$ This study investigated the variability of the frontal plane forefoot-torearfoot relationship in neutral subtalar joint casting. The results of this study show that there is no difference among the different groups of clinicians, indicating that the level of experience of the examiner did not affect the accuracy of the negative plaster cast in capturing the forefoot-to-rearfoot relationship. Although there was no statistical difference in the means or variability among the groups, the range of the forefoot-to-rearfoot relationships across all groups was $16.5^{\circ}\left(10.0^{\circ}\right.$ everted to $6.5^{\circ}$ inverted), which is substantial. This could be viewed with concern if it is assumed that the cast of the foot is supposed to accurately capture the true forefoot-to-rearfoot relationship if the functional foot orthosis is to allow the foot to function about the assumed neutral position of the subtalar joint. ${ }^{4,6,7}$

The variability reported here seems to be higher 
than the standard deviations reported by McPoil et $\mathrm{al}^{2}$ comparing the forefoot-to-rearfoot relationship in casts taken prone, supine, and semiweightbearing for 11 subjects. In that study, one experienced examiner took all of the casts. The variability reported here is consistent with the finding of Pierrynowski et $\mathrm{al}^{11}$ of a high degree of difference among eight experienced clinicians in the accuracy of placing the foot in its subtalar joint neutral position. In the context of their finding, it would be expected that casting of the foot based on use of the subtalar joint neutral position would be subject to similar variability. Although it is still most widely used as a reference position, the assumption that the subtalar joint functions about its defined neutral position has been questioned. ${ }^{12}$

The original concept of putting the subtalar joint in its neutral position and maximally pronating the midtarsal joint longitudinal and oblique axes is flawed because it is kinematically impossible for the midtarsal joint to have motion about these two axes. ${ }^{13}$ Despite the current lack of support for the two axes midtarsal joint model, ${ }^{14}$ it is helpful to adopt the construct of the model as a convenient theoretical fiction to facilitate understanding of foot function. ${ }^{15}$ It is assumed for casting that the foot should be pronated about both the putative longitudinal and oblique axes to capture the true osseous forefoot-to-rearfoot position. In this study, five casts were taken with the foot assumed to be maximally pronated about the longitudinal axis (by applying a dorsiflexory force to the lateral side of the forefoot) and pronated about the oblique axis (by applying an abductory force to the forefoot) while holding the subtalar joint in its neutral position. Another five casts were taken with the foot again maximally pronated about the longitudinal axis (by applying a dorsiflexory force to the lateral side of the forefoot) and supinated about the oblique axis (by applying an adductory force to the forefoot). If the forefoot was supinated about the oblique axis of the midtarsal joint, it would be assumed that the forefoot would be inverted relative to the rearfoot compared with a cast of the foot that was pronated about the oblique axis of the midtarsal joint. This study did not find any difference in the frontal plane forefoot-to-rearfoot relationship between the two midtarsal joint positions. It is unclear why no difference was found; the assumption of a theoretical difference may be incorrect.

In usual clinical practice, clinicians evaluate the cast before deciding to use it for a foot orthosis or to take it again. ${ }^{16}$ A shortcoming of this study is that the clinicians taking the cast were not permitted to do this. If clinicians were permitted to take a second cast and to decide which more accurately represent- ed the foot based on a structured evaluation, the variability may have been less.

\section{Conclusion}

The results of this study show that there is wide variability in the frontal plane forefoot-to-rearfoot relationship in neutral-position casting of the foot and that there is no difference between experienced and inexperienced clinicians. Despite this variability in casting and the wide use of functional foot orthoses made from these casts, results of outcome studies ${ }^{17}$ have clearly shown that functional foot orthoses are effective in providing symptomatic relief, raising the question of the necessity of an accurate cast. It also raises the possibility of functional foot orthoses being effective for reasons other than the assumed importance of the subtalar joint being in its neutral position and the midtarsal joint being in its defined locked position at midstance.

\section{References}

1. Foulston J, LoRd M, West S: Changes in plantar surface shape induced by corrective forefoot eversion. Clin Biomech 5: 229, 1990.

2. McPoll TG, Schuit D, Knecht HG: Comparison of three methods used to obtain a neutral plaster foot impression. Phys Ther 69: 448, 1989.

3. Cox RA, IngRANDo CR, PARK SC, ET AL: Comparison of forefoot varus measurements obtained from non-weightbearing, partial weightbearing, and full weightbearing orthotic casting techniques [abstract]. J Orthop Sports Phys Ther 29: A5, 1999.

4. Losito JM: "Impression Casting Techniques," in Clinical Biomechanics of the Lower Extremity, ed by RL Valmassy, p 279, CV Mosby, St Louis, 1996.

5. Root ML, Weed JH, Orien WP: Neutral Position Casting Techniques, Clinical Biomechanics Corp, Los Angeles, 1971.

6. Philps JW: The Functional Foot Orthoses, 2nd Ed, Churchill Livingstone, Edinburgh, 1992.

7. Anthony R: Manufacture and Use of the Functional Foot Orthoses, Karger, Basel, 1991.

8. Root ML, ORIen WP, Weed JH: Normal and Abnormal Function of the Foot, Clinical Biomechanics Corp, Los Angeles, 1977.

9. Shrout PE, Fleiss JL: Intraclass correlation: uses in assessing rater reliability. Psychol Bull 86: 420, 1979.

10. VALMASSY RL: Advantages and disadvantages of various casting techniques. JAPA 69: 707, 1979.

11. Pierrynowski MR, Smith SB, Mlynarczyk JH: Proficiency of foot care specialists to place the rearfoot at subtalar neutral. JAPMA 86: 217, 1996.

12. MCPoil TG, CoRnwall MW: The relationship between subtalar joint neutral position and rearfoot motion during walking. Foot Ankle 15: 141, 1994.

13. Nester CJ, Findlow A, Bowker P: Scientific approach to the axis of rotation at the midtarsal joint. JAPMA 91: 68, 2001. 
14. KeEnAn AM: "Understanding Midtarsal Joint Function: Fact and Fallacy," in Proceedings of the 17th Australian Podiatry Conference, ed by AM Keenan, HB Menz, p 107, Australian Podiatry Council, Melbourne, Australia, 1996.

15. PAYNE CB: The role of theory in understanding the mid- tarsal joint. JAPMA 90: 377, 2000.

16. Ross AS, Jones LJ: Non-weightbearing negative cast evaluation. JAPA 72: 634, 1982.

17. OLSEN WR: "Orthotic Materials," in Clinical Biomechanics of the Lower Extremity, ed by RL Valmassy, p 307, CV Mosby, St Louis, 1996. 